\title{
All-fiber Yb-doped Laser Mode-locked by Nanotubes
}

\author{
Z. Zhang ${ }^{1}$, D. Popa ${ }^{2}$, Z. Sun ${ }^{2}$, T. Hasan ${ }^{2}$, A. C. Ferrari ${ }^{2}$ and F. Ö. Ilday ${ }^{1}$ \\ ${ }^{1}$ Department of Physics, Bilkent University, 06800 Ankara, Turkey \\ ${ }^{2}$ Department of Engineering, University of Cambridge, CB3 OFA Cambridge, UK
}

Single-wall carbon nanotubes (SWNTs) and graphene have emerged as promising saturable absorbers (SAs), due to their broad operation bandwidth and fast recovery times [1-3]. However, Yb-doped fiber lasers mode-locked using CNT and graphene SAs have generated relatively long pulses. All-fiber cavity designs are highly favored for their environmental robustness. Here, we demonstrate an all-fiber Yb-doped laser based on a SWNT saturable absorber, which allows generation of 8.7 ps-long pulses, externally compressed to $118 \mathrm{fs}$. To the best of our knowledge, these are the shortest pulses obtained with SWNT SAs from a Yb-doped fiber laser.

The experimental setup is shown in Fig.1(a). The gain section is a $1.88 \mathrm{~m}$-long Yb-doped fiber with 35.5 $\mathrm{fs}^{2} / \mathrm{mm}$ group velocity dispersion, followed by a $20 \%$ output coupler and an inline isolator. A $2.1 \mathrm{~m}$-long solidcore photonic crystal fiber (PCF) is employed for dispersion management. A scanning electron microscope image of the PCF is shown in Fig. 1(a) bottom. The PCF has a dispersion of $-46.3 \mathrm{fs}^{2} / \mathrm{mm}$ at $1.04 \mu \mathrm{m}$. All other fibers used in the cavity, are standard single-mode fiber (SMF). The net dispersion of the cavity is estimated to be 0.014 $\mathrm{ps}^{2}$. The SA is fabricated as described in [2], and integrated into the cavity between a pair of fiber connectors.
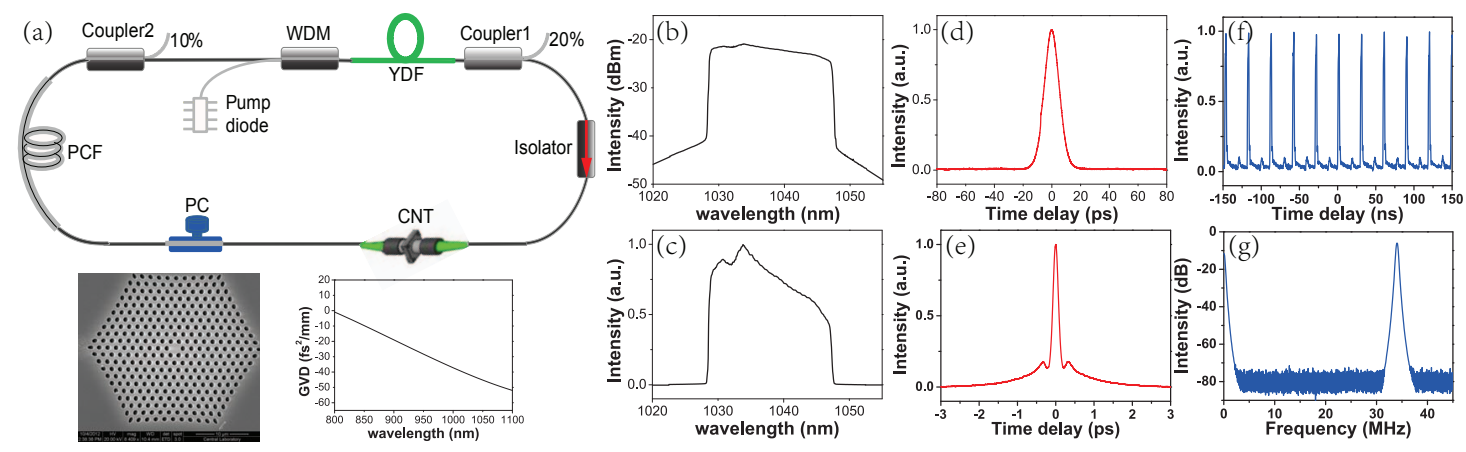

Fig. 1 (a) Schematic of the laser. Optical spectra of output pulse on (b) logarithmic scale and (c) linear scale. Autocorrelation traces before (d) and after (e) dechirping. (f) Pulse train. (g) RF spectrum.

Figures 1(b-g) show the typical output performances at a pump power of $92 \mathrm{~mW}$. Figure 1(b) and (c) show the output spectrum on logarithmic and linear scales, respectively. The spectrum has a $3 \mathrm{~dB}$ bandwidth of $17.6 \mathrm{~nm}$. Figures 1(d) and (e) present autocorrelation traces before and after external pulse compression. The duration of the chirped pulses is $8.7 \mathrm{ps}$. Using a grating pair with anomalous dispersion of $-0.25 \mathrm{ps}^{2}$, a dechirped $118 \mathrm{fs}$ pulse is obtained. This indicates that intracavity pulses are always chirped along the whole laser cavity. Fig. 1(f) shows the output pulse train. The fundamental repetition rate of the laser is $34 \mathrm{MHz}$. The RF spectrum (Fig. 1(g)) has $\sim 70$ $\mathrm{dB}$ signal to noise ratio. The maximal output power is $\sim 3.5 \mathrm{~mW}$. The peak power of the pulses within the PCF is much lower than the limit set by nonlinear processes, based on our calculations and experiments on a similar cavity, which used nonlinear polarization evolution instead of CNT as SA. Therefore, we conclude that the pulse energy is limited by overdriving of the CNT instead.

In conclusion, we demonstrated ultrashort pulse generation from an all-fiber Yb-doped fiber laser mode-locked with a SWNT saturable absorber. The output pulses are compressed to $118 \mathrm{fs}$ outside the laser cavity. A PCF is utilized to create a dispersion map with net normal dispersion and the pulses have large positive chirp at all points in the cavity. This laser exhibits elements of dissipative soliton formation [4], which has great potential for high-energy pulse generation, despite the presence of a dispersion map. The effect of the dispersion map remains weak due to the small nonlinearity experienced by the pulse, which is clamped in power by over saturation of the SWNT. However, additional work is needed to definitively identify the pulse evolution within the cavity.

\section{References}

[1] F. Bonaccorso, Z. Sun, T. Hasan, and A. C. Ferrari, "Graphene photonics and optoelectronics," Nat. Photon. 4, 611 (2010).

[2] T. Hasan, Z. Sun, F. Wang, F. Bonaccorso, P. H. Tan, A. G. Rozhin, and Ferrari A C, "Nanotube-polymer composites for ultrafast photonics," Adv. Mater. 21, 3874 (2009).

[3] D. Popa, Z. Sun, T. Hasan, W. B. Cho, F. Wang, F. Torrisi, and A. C. Ferrari, "74-fs nanotube-mode-locked fiber laser," Appl. Phys. Lett. 101, 153107 (2012).

[4] P. Grelu, N. Akhmediev, ’Dissipative solitons for mode-locked lasers," Nat. Photon. 6, 84 (2012). 